\title{
Chemical tagging of Stellar streams: Origin Scenarios
}

\author{
Ramya Pozhath*ं \\ Indian Institute of Astrophysics, Bangalore, India \\ E-mail: ramyap@iiap.res.in

\section{Bacham Eswar Reddy} \\ Indian Institute of Astrophysics, Bangalore, India \\ E-mail: ereddy@iiap.res.in
}

\begin{abstract}
Stellar streams are kinematically coherent groups of stars, scattered all over the sky, and hence gravitationally unbound. They appear as over-densities in the velocity space. Open clusters are groups of coeval, chemically homogeneous stars, gravitationally bound with each other. The striking similarity between stellar streams and open clusters is that, both are kinematically coherent groups. It has been long thought that stellar streams formed as a result of open cluster disruption, the dispersed members having retained the parental cluster kinematics, and are the intermediate phase between open clusters and field stars in the Galaxy. While many of the proposed stellar streams in the literature share the motion of well known open clusters, some streams do exist without any defining clusters. Here, we discuss streams such as Arcturus, AF06, Hercules, Hyades-Pleiades and Sirius based on abundance results. The significant result of this study is that none of the streams show chemical homogeneity, and the abundance pattern imply member stars may not have originated solely from their corresponding defining clusters
\end{abstract}

XIII Nuclei in the Cosmos,

7-11 July, 2014

Debrecen, Hungary

* Speaker.

${ }^{\dagger}$ A footnote may follow. 


\section{Introduction}

In general, field stars within the disc or in the halo are expected to exhibit random motion such as three dimensional Schwarzschild velocity distribution. Contrary to this general expectation, it has been known in the literature for more than a century, there exist stars that form clumps in the velocity space sharing common motion. The kinematically coherent groups are known as moving groups or stellar streams. Stellar streams are defined as the tight clumps or over-densities of stars present in the velocity space over and above the large scale structure that Schwarzschild distribution can reproduce in the solar neighborhood.

The concept of stellar streams has been around in the literature for long, but their origin and evolution within the Galaxy remained very poorly understood. There are three principal hypothesis for the existence of the moving groups: a) cluster disruption, b) satellite accretion, c) dynamical perturbations. According to cluster disruption hypothesis, open clusters while orbiting around the Galactic center get disrupted due to encounters with the giant molecular clouds. The dispersed cluster members retain parental kinematics, thus forming moving groups in the Galaxy. As per the satellite accretion scenario, the Milky Way accretes its dwarf satellite galaxies and the debris of such accreted satellites remain in the Galaxy as stellar streams or moving groups. Finally, dynamical perturbation scenario says that the dynamical perturbations introduced by non-axisymmetric components of the Galaxy such as the bar and/or spiral arms or the merging satellite galaxies produce moving groups.

In this, we studied a few identified moving groups based on chemical tagging of their member stars. The chemical tagging (Freeman \& Bland-Hawthorn 2002) is to understand stars' past history as their chemistry remains unaltered, to a large extent, over their life times. We performed abundance analysis of member stars of a few moving groups in search of clues to their origin. Understanding of the moving groups has far reaching significance in decoding the chronological evolution of the Galaxy. We chose five well defined moving groups for this study. Of which, two moving groups (Arcturus and AF06) belong to the thick disc and three (Hercules, Hyades-Pleiades and Sirius moving groups) belong to the thin disc component of the Galaxy. A brief description of each of the streams is given below.

\section{Streams in the Galactic thin disc}

Hercules stream, Hyades-Pleiades stream and Sirius stream :

58 Hercules stream members, 34 Hyades-Pleiades members and 33 Sirius stream members were chosen from Famaey et. al. (2005), for the detailed analysis. The kinematics of Hercules and Sirius stream are : $\mathrm{U}=-42.13 \pm 1.95, \mathrm{~V}=-51.64 \pm 1.07, \mathrm{~W}=-8.06 \pm 1.30 \mathrm{kms}^{-1}$ and $\mathrm{U}=6.53 \pm$ $1.93, \mathrm{~V}=3.96 \pm 0.67, \mathrm{~W}=-5.80 \pm 1.15 \mathrm{kms}^{-1}$ respectively, while Hyades and Pleiades streams could not be resolved and they appeared as a single clump at $\mathrm{U}=-30.34 \pm 1.54, \mathrm{~V}=-20.27 \pm$ $0.62, \mathrm{~W}=-4.82 \pm 0.80 \mathrm{kms}^{-1}$. All the sample members are $\mathrm{K}$ and $\mathrm{M}$ giants.

High resolution spectra of the stream members were observed using 2.7 m Harlan J. Smith Telescope of McDonald Observatory, Texas and $2.3 \mathrm{~m}$ Vainu Bappu Telescope of Vainu Bappu Observatory, Kavalur. Abundances of elements belonging to various groups (light, alpha, iron and heavy elements) have been estimated using the equivalent widths measured from the spec- 
Hercules
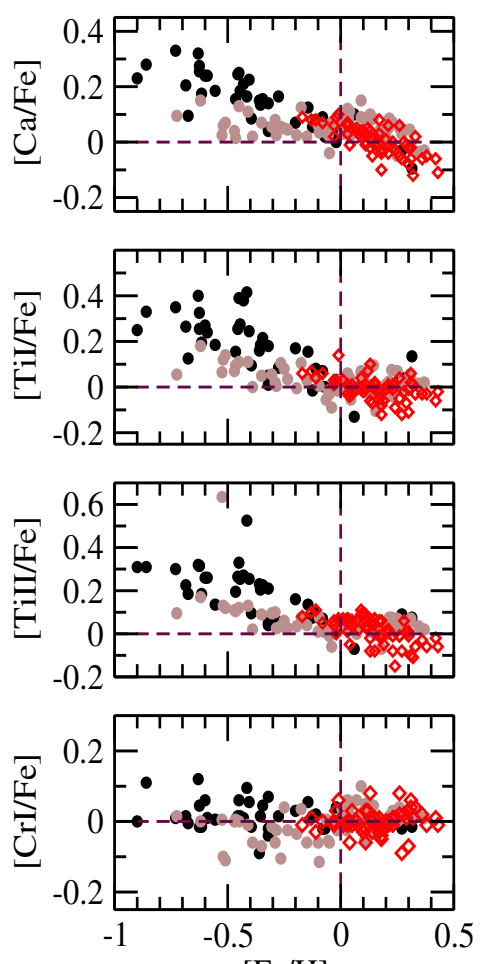

$[\mathrm{Fe} / \mathrm{H}]$
Hyades-Pleiades
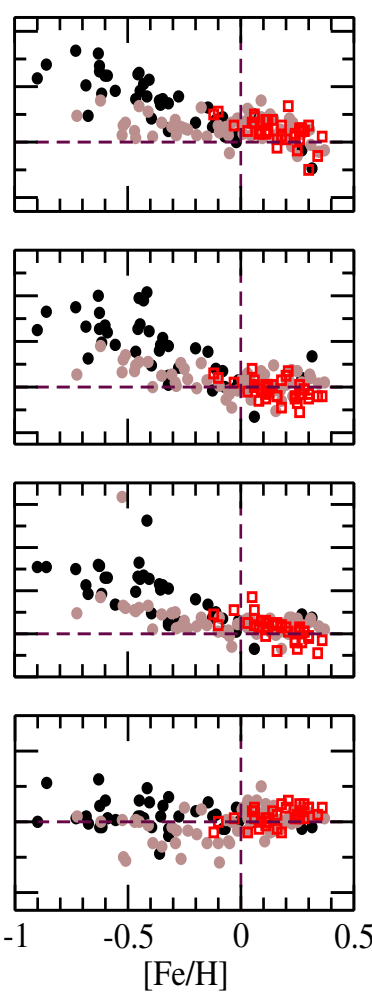

Sirius
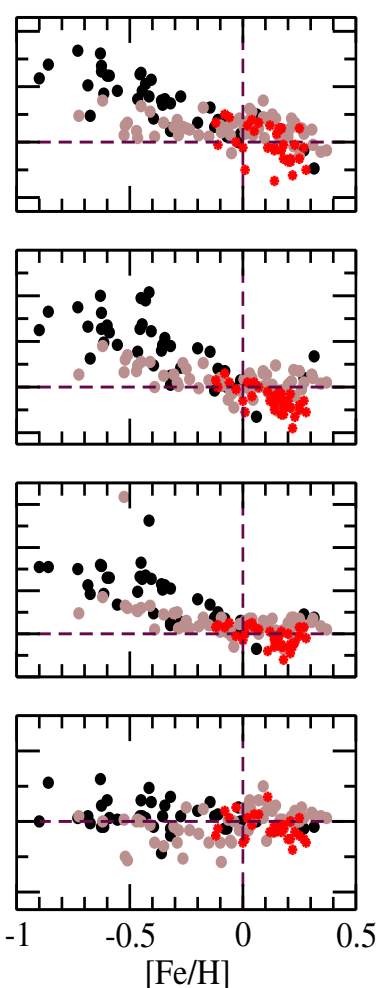

Figure 1: The abundance pattern of the streams (Red square) compared with that of Galactic thin disc (brown circle) and thick disc (black circle) taken from Bensby et. al. (2005)

tra, stellar model atmospheres (Kurucz 1998) and spectrum analysis code MOOG (Sneden 1973). Abundances of a few representative elements against $[\mathrm{Fe} / \mathrm{H}]$ are shown in Figure 1 along with abundances of sample stars of Galactic thin and thick disc components taken from Bensby et. al. (2005). Hercules stream has a large range in metallicity, $[\mathrm{Fe} / \mathrm{H}]=-0.17$ dex to +0.43 dex, with a mean of $+0.15 \pm 0.14$ dex which is typical of thin disc stars. The metallicity of most of the members stars of Hyades-Pleiades stream is super solar ranging from $[\mathrm{Fe} / \mathrm{H}]=-0.12 \mathrm{dex}+0.36 \mathrm{dex}$. To test the possibility that whether the Hyades stream sample contains stars evaporated from the Hyades cluster or not, we scrutinized and compared elemental abundances of the moving group with the observed members of Hyades open cluster. We could find three member stars (10\% of the entire sample) of age and abundance pattern very similar to those of Hyades open cluster.Similarly, Sirius stream members yielded a mean metallicity of $[\mathrm{Fe} / \mathrm{H}] \sim 0.12$ dex. Agreeing with their metal-rich stars, member stars of the three strems are found to be young with ages spanning from few Myrs to about 4 Gyr. Both the metallicity and ages indicate member stars are drawn from the thin disc population. The age distribution and the $(\mathrm{B}-\mathrm{V})-\mathrm{M}_{v}$ plane with isochrones overplotted for the three streams are shown in Figure 2. 

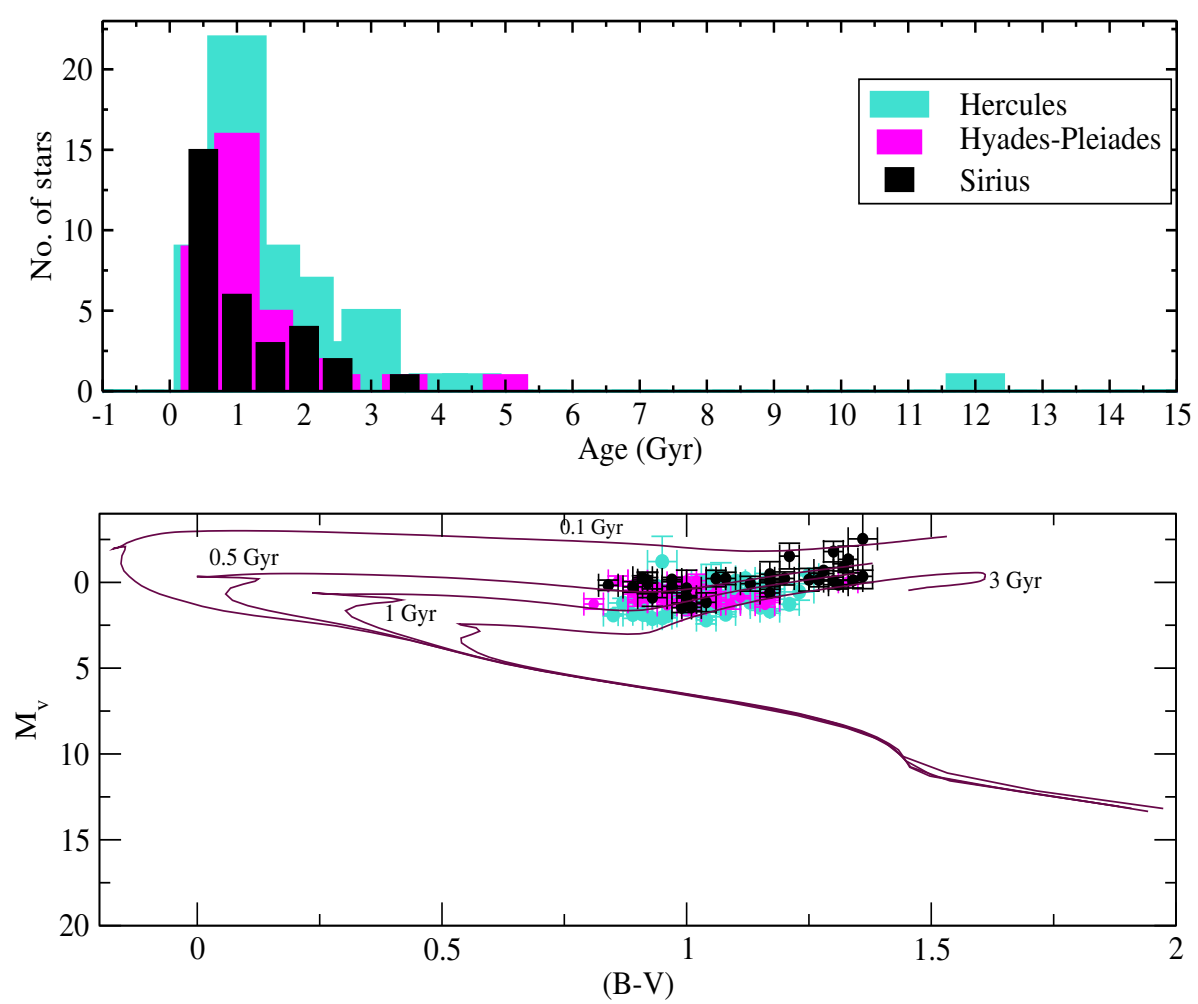

Figure 2: The age distribution of the three streams (top one) and the three streams on (B-V) - $\mathrm{M}_{v}$ plane (bottom one) with isochrones overplotted

\section{Streams in the Galactic thick disc}

Arcturus and AF06 stream :

A sample of total 40 main sequence stars for the two thick disc streams has been chosen for chemical tagging study : 18 for Arcturus stream and 22 for AF06 stream. Sample stars are F and G subdwarfs taken from the study of Arifyanto \& Fuchs (2006).

Detailed abundance analysis of both the stream members has been performed and the abundances of verious elements were estimated. Abundance patterns of $\alpha$ elements are given in Figure 3 as $[\alpha / \mathrm{Fe}]$ versus $[\mathrm{Fe} / \mathrm{H}]$ along with Galactic thick disc pattern taken from Reddy et. al. (2006). From the figure, it is clear that Arcturus stream and AF06 are metal poor with $[\mathrm{Fe} / \mathrm{H}]$ ranging from -1.69 dex to +0.22 dex, and they do not have a unique abundance pattern, but just follow the Galactic thick disc abundance trends. The ages estimated using $\mathrm{Y}^{2}$ isochrones show that both the stream members are very old (10-14 Gyr) similar to that of the thick disc component.

\section{Origin of the streams}

Member stars of Hercules stream, Arcturus stream and AF06 stream show large range in metallicity and age which are relatively much higher compared to any typical surviving open clusters which show very little star-to-star dispersion. Similarly, large range in metallicity ( $0.4 \mathrm{dex})$ and ages (4 Gyr) for Sirius stream imply chemical inhomogeneity and noncoeval nature of the member 

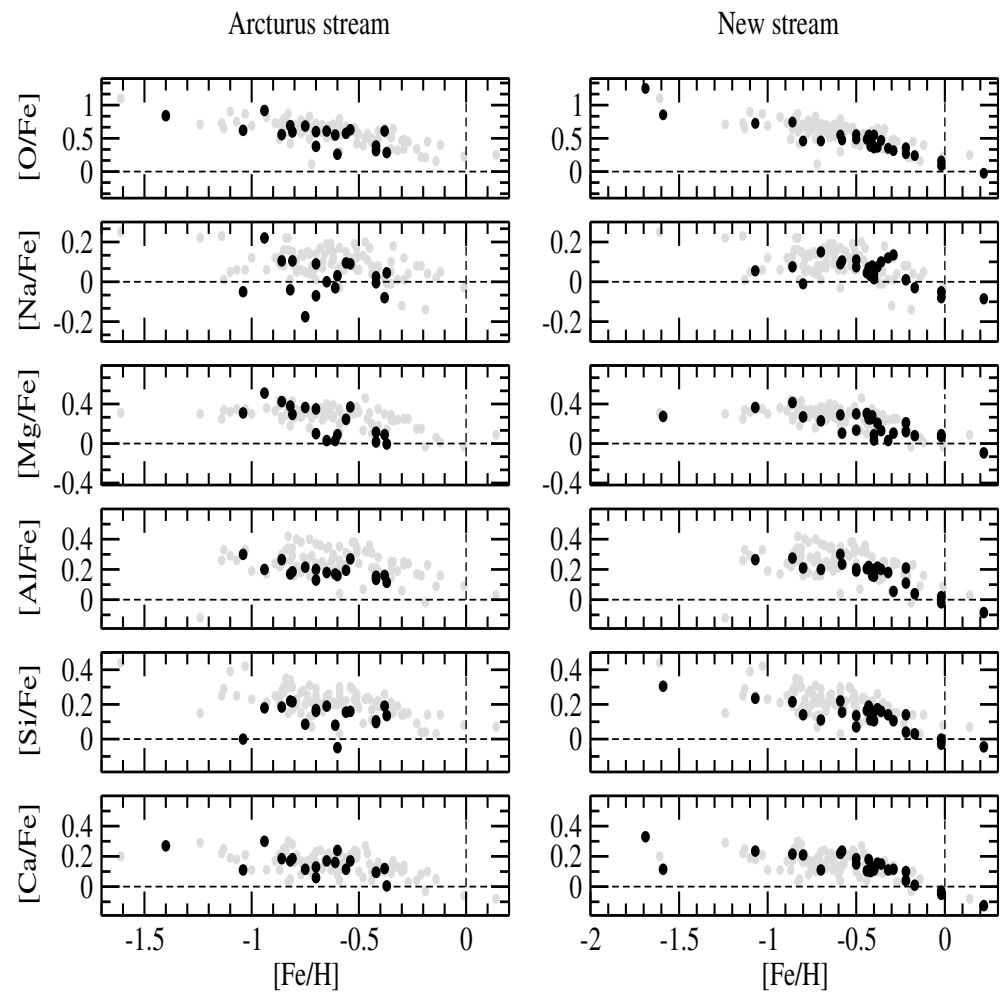

Figure 3: The $[\alpha / \mathrm{Fe}]$ versus $[\mathrm{Fe} / \mathrm{H}]$ plot. Black and Grey symbols represent the stream members and the Galactic thick disc stars respectively

stars. This leads to the conclusion that members of Hercules Arcturus, AF06 and Sirius stream are groups of stars share only kinematics but not chemistry or coevality. So open cluster dispersion may not be the origin for these streams. However, a fraction of stars of Hyades stream possibly originated from the disrupted Hyades cluster as few stream members share the chemistry of the open cluster. We also rule out the possibility that these stars came from merged dwarf spheroidals as none of the streams abundance trensd match with any of the known dwarf spheroidals (see Ramya et al. 2012 for more details). Thus, our results indicate that these streams formed in-situ due to dynamical effects caused by Galactic bar (Navarro et al. 2004).

The major findings of this study are a) None of the five streams studied here show chemical homogeneity and coevality, the defining properties of spatially coherent groups. b) None of the streams studied here show any sort of similarity with the dwarf satellite galaxies of the Milky Way galaxy. c) Abundance patterns are indistinguishable from that of the background Galactic disc (thin or thick disc) field stars. d) A fraction of member stars of the moving groups (in particular Sirius, Hyades) seems to be originated from the cluster dispersion.

\section{References}

[1] Arifyanto M. I., Fuchs B., 2006, A\&A, 449, 533

[2] Bensby T., Feltzing S., Lundström I., Ilyin I., 2005, A\&A, 433, 185 
[3] Freeman K., Bland-Hawthorn J., 2002, ARA\&A, 40, 487

[4] Famaey B., Jorissen A., Luri X., Mayor M., Udry S., Dejonghe H., Turon C., 2005, A\&A, 430, 165

[5] Kurucz R.L., 1998, http://kurucz.harvard.edu/ (online data)

[6] Navarro J. F., Helmi A., Freeman K. C., 2004, ApJL, 601, L43

[7] Ramya P., Reddy B. E., Lambert D. L., 2012, MNRAS, 425, 3188

[8] Reddy B. E., Lambert D. L., Allende Prieto C., 2006, MNRAS, 367, 1329

[9] Sneden C., 1973, PhD Thesis, University of Texas, Austin 